\title{
Ultrashort pulse generation based on two coupled helical gyro-TWTs
}

\author{
M.N. Vilkov, N.S. Ginzburg, G.G. Denisov, I.V. Zotova, A.S. Sergeev, A.M. Malkin, \\ S.V. Samsonov and S.V. Mishakin \\ Institute of Applied Physics RAS, Nizhny Novgorod, Russia, vilkovmn@ipfran.ru
}

In laser physics, there exists a well-known principle for production of optical ultrashort pulses (USP), based on the effect of passive mode-locking [1], which is achieved by incorporating a saturable absorber into the laser resonator. As it was shown in [2], a similar method of ultrashort pulse generation can be realized in a twosection microwave oscillator consisting of an amplifier and a nonlinear absorber in the feedback loop.

The basic requirement on the amplifying unit is a broad frequency bandwidth that is needed for effective amplification of ultrashort microwave pulses. In the millimeter wave band, it is attractive to utilize a novel type of gyro-TWTs with helically corrugated waveguides operating at harmonics of epy gyrofrequency [3, 4]. Such devices have a number of unique characteristics, including an extremely broad frequency bandwidth up to $20 \%$.

The key problem for development of mode-locked electron oscillators is realization of a nonlinear absorber which is applicable to the microwave frequency band and suitable for operation at the high-power level. In this paper we suggest to use for this purpose the additional section with independent electron beam where interaction also takes place in a helically corrugated waveguide at a harmonic of the gyrofrequency. In such a system saturable absorption realizes for magnetic field slightly exceeding the resonance one. In fact this area corresponds to the so-called Kompfner dip regime, which was originally studied for Cherenkov TWTs [5]. We demonstrate that after proper matching of parameters, the Kompfner dip regime with saturation of absorption can be realized also in a gyro-TWT with a helically corrugated waveguide.

The scheme of the considered microwave USP oscillator with passive mode-locking is presented in Fig. 1. This oscillator includes two gyro-TWT sections with a helical corrugation, one of which is used as an amplifying unit, while another operates in the regime of nonlinear absorption.

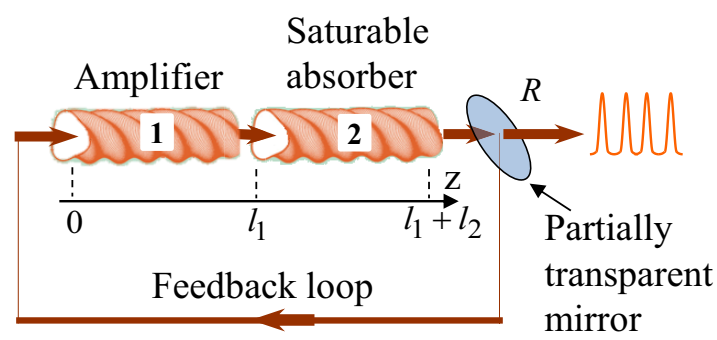

Fig. 1. Scheme of a mode-locked microwave oscillator consisting of two coupled helical gyro-TWTs

Multi-fold helical corrugation provides the coupling of two partial $\mathrm{TE}_{\mathrm{m}, \mathrm{n}}$ modes: a near-cutoff mode $\mathrm{A}$ and a far-from-cutoff traveling mode B. Correspondingly, electron-wave interaction in both sections is described by the equations [6]:

$$
\begin{aligned}
& \frac{\partial^{2} a}{\partial z^{2}}-2 i \frac{\kappa_{A}}{c} \frac{\partial a}{\partial t}-2 \sigma \kappa_{A}^{2} b= \\
& =i \frac{4 e I_{b}}{\pi m c^{3}} \frac{\kappa_{A}^{2}}{\beta_{\| 0} \sqrt{N_{A}}} \frac{s^{s}}{2^{s} s !} \int_{0}^{2 \pi} p_{+}^{s} d \theta_{0}, \\
& \frac{\partial b}{\partial z}+\frac{1}{V_{g r}} \frac{\partial b}{\partial t}-i\left(\bar{h}-h_{0}\right) b-i \frac{\kappa_{A}^{2}}{h_{0}} \sigma a=0, \\
& \frac{\partial p_{+}}{\partial z}+\frac{1}{V_{\| 0}} \frac{\partial p_{+}}{\partial t}+i \frac{\kappa_{A}}{2 \beta_{\| 0}} \frac{p_{+}}{s}\left(\Delta+\left|p_{+}\right|^{2}-\beta_{\perp 0}^{2}\right)= \\
& =\frac{s^{s}}{2^{s} s ! \beta_{\| 0} \sqrt{N_{A}}} a\left(p_{+}^{*}\right)^{s-1} .
\end{aligned}
$$

Here $p_{+}$is the transverse electron momentum, $a=e A \sqrt{N_{A}} / m c^{2} \kappa_{A}$ and $b=e B \sqrt{N_{B}} / m c^{2} \kappa_{B}$ are the normalized amplitudes of partial waves, $\sigma=\left(\tilde{r} / 2 r_{0}\right)\left(v_{B}^{2}-m_{A} m_{B}\right) / \sqrt{\left(v_{A}^{2}-m_{A}^{2}\right)\left(v_{B}^{2}-m_{B}^{2}\right)}$ is the wave coupling parameter [3] proportional to the corrugation depth $\tilde{r}, r_{0}$ is the mean radius of a corrugated waveguide, $N_{A}$ and $N_{B}$ are the dimensionless norms of the partial waves, $\quad V_{g r}=h_{0} c / \kappa_{A}$ is the group velocity of the traveling wave $B, V_{\| 0}=\beta_{\| 0} c$ is the longitudinal velocity of electrons, $I_{b}$ is the beam current, $\Delta=2\left(\omega_{A}-s \omega_{H}\right) / \omega_{A}$ is the cyclotron resonance detuning, $h_{0}=h_{B}\left(\omega_{A}\right)$ is the longitudinal wave number of the traveling wave $B$ at the frequency $\omega_{A}, \kappa_{A, B}=\mathrm{v}_{A, B} / r_{0}$ are transverse wave numbers for partial waves $A$ and $B$, correspondingly, $\bar{h}=2 \pi / d, d$ is the corrugation period, $s$ is the electron cyclotron harmonic number.

We assume that the amplifying and absorbing sections are coupled via the traveling partial wave B In accordance with the scheme shown in Fig. 1 boundary conditions can be presented in the form

$$
b_{2}\left(t, l_{1}\right)=b_{1}\left(t, l_{1}\right), b_{1}(t, 0)=R b_{2}\left(t-T, l_{1}+l_{2}\right)
$$

where $R<1$ is the reflection coefficient.

Simulations were performed for parameters the experimentally realized Ka-band helical gyro-TWT [4]: the particles energy of 50-70 keV, the total current of 2-10 A, the pitch-factor of $0.8-1.2$. For this amplifier the second helical gyro-TWT section was synthesized (the particles energy is $54 \mathrm{keV}$, the total current is $6 \mathrm{~A}$, the pitch-factor is 0.53 ) which operates like a saturable absorber and provides USPs generation. Both TWTs have three-fold helically corrugated waveguides for resonant coupling of the near-cutoff $\mathrm{TE}_{2,1}$ and counter-rotating traveling $\mathrm{TE}_{1,1}$ mode. The indicated modes are selectively excited by an axis-encircling beam at the second cyclotron harmonic $(s=2)$. 
In the amplifying section in contrast to the gyroTWT [4] we choose parameters of corrugation in such a way that the intersection between the beam line and the dispersion curve of the normal mode is provided (Fig. 2a). In this case the wave group velocity substantially differs from the axial velocity of the electrons. As a result due to slippage of em pulse over the electrons it effectively accumulates the energy from different fractions of the electron beam. On the contrary, in the absorbing section, for minimization of the absorber relaxation time the grazing incidence of dispersion characteristics of the electron beam and the normal wave of a corrugated waveguide is beneficial (Fig. $2 b$ ).
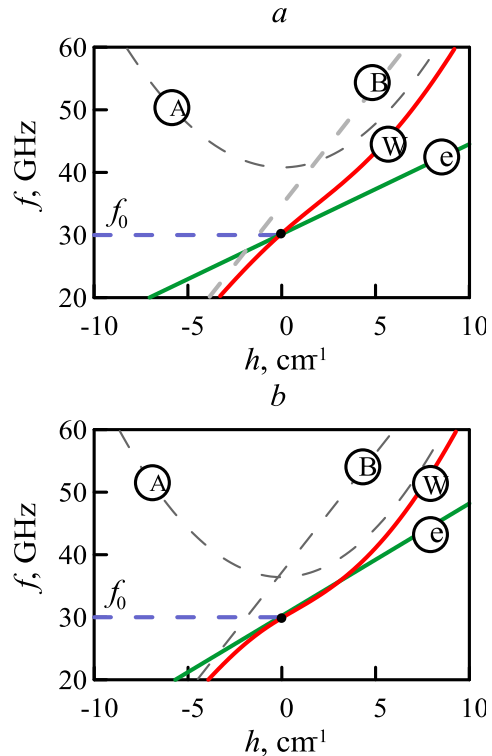

Fig. 2. Dispersion diagrams of the partial $(A, B)$, normal $(W)$ and electron cyclotron (e) waves in the amplifier (a) and absorbing section (b) for operating frequency $30 \mathrm{GHz}$

Simulations show that the maximum stability and reproducibility of generated pulses is achieved in a high excitation mode of the considered oscillator, when generation starts after initiation by a sufficiently large initial pulse. In the absence of the source of an external pulse, we can use the startup scenario of USP generation with delayed switching of the absorbing section with respect to the amplifying section. In this case, at the first stage only the amplifier is switched on and, consequently, chaotic generation develops from small initial noises (Fig. 3b). Then the absorbing section is switched on and, as a result, the chaotic regime of generation transforms to the periodical regime of USPs generation (Fig. 3c). For chosen parameters the duration of the generated pulses is $0.2 \mathrm{~ns}$ with distance between them of $1 \mathrm{~ns}$. Peak power of pulses achieves $400 \mathrm{~kW}$, that (due to slippage in the amplifying section) exceeds in 2 times the maximum output power level in a helical gyro-TWT [4] with the similar parameters. Currently this type of USP oscillator is under development at the Institute of Applied Physics RAS. $a$
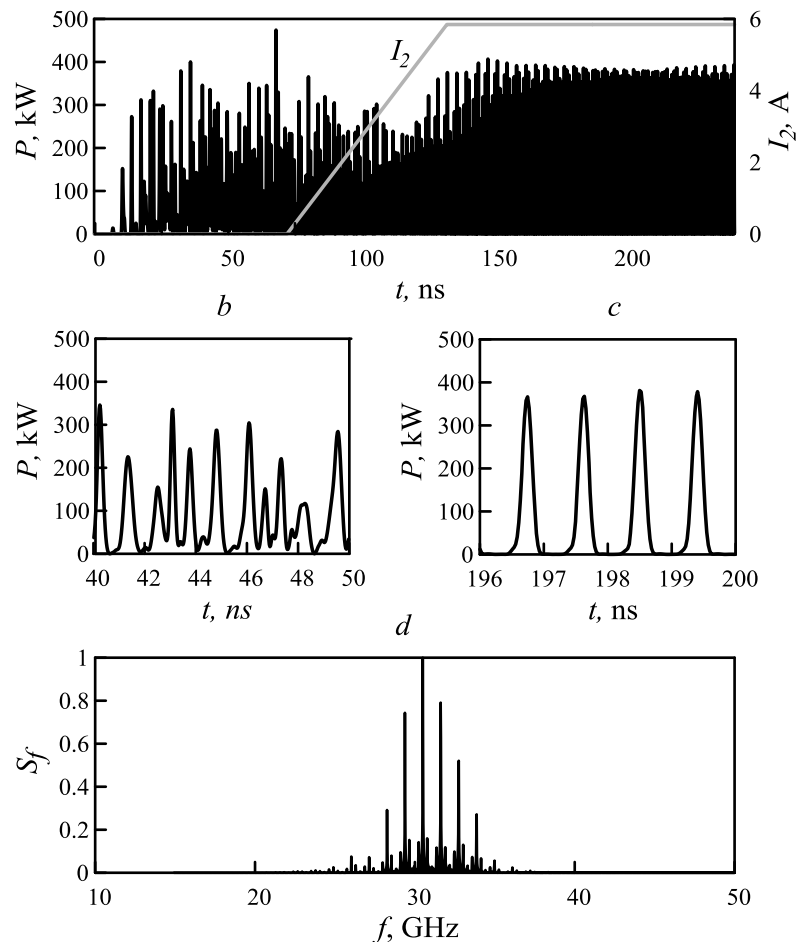

Fig. 3. Setting the USP generation regime when the absorbing section switches on with a delay relative to the amplifying section: temporal dependence of the output power and the current $I_{2}$ in the absorber (a), detailed profiles of the generated signal on the stage of developing a chaotic self-modulation (b) and on the stage of the ultrashort pulses generation after the switching of the absorber (c), the radiation spectrum (d)

This work was supported by Russian Science Foundation (RSCF), grant No. 16-42-01078.

\section{References}

1. Haus H.A. Mode-Locking of Lasers // IEEE J. of Select. Topics in Quant. Electron. 2000. V. 6, No. 6. P. 1173-1185.

2. Ginzburg N.S., Denisov G.G., Vilkov M.N. et al. Generation of "gigantic" ultra-short microwave pulses based on passive mode-locking effect in electron oscillators with saturable absorber in the feedback loop // Phys. Plasmas. 2016. V. 23. P. 050702-1-4.

3. Denisov G.G., Bratman V.L., Cross A.W. et al. Gyrotron Traveling Wave Amplifier with a Helical Interaction Waveguide // Phys. Rev. Lett. 1998. V. 81, No. 25. P. 5680-5683.

4. Samsonov S.V., Gachev I.G., Denisov G.G. et al. Kaband gyrotron traveling-wave tubes with the highest continuous-wave and average power // IEEE Trans. Electron Devices. 2014. V. 61, No. 12. P. 4264-4267.

5. Kompfner R. On the operation of the traveling wave tube at low level // J. Brit. IRE. 1950. V. 10, No 8-9. P. 283-289.

6. Ginzburg N.S., Zotova I.V., Sergeev A.S., Zaslavsky $V . Y u$. et al. Mechanisms of amplification of ultrashort electromagnetic pulses in gyrotron traveling wave tube with helically corrugated waveguide // Phys. Plasmas. 2015. V. 22 P. 113111-1-7. 